\title{
\#INDIGNACIÓN EN ANDALUCÍA: ORIGEN Y ANDADURA INICIAL DEL MOVIMIENTO 15-M
}

\section{\#Outrage in Andalusia: origin and initial steps of the 15-m movement}

José Peña Ramos**

Recibido: 19 de octubre de 2012. Aprobado: 8 de abril de 2013.

\section{RESUMEN}

El presente artículo exploratorio pretende realizar una aproximación inicial al origen, la evolución inmediata y los efectos del Movimiento 15-M, concretamente en Andalucía. En primer lugar, se establece un breve marco teórico-conceptual de referencia. En segundo lugar, se contextualiza este movimiento en el plano internacional, y se establecen sus principales características. En tercer lugar, se aborda el movimiento en el ámbito andaluz. En cuarto lugar, se analiza su impacto sobre la opinión pública andaluza. Y finalmente se aportan unas conclusiones.

PALABRAS CLAVES: Indignados, Andalucía, movimientos sociales.

\begin{abstract}
This exploratory article aims to make an initial analysis of the origins, immediate evolution and the effects of the 15-M Movement, particularly in Andalusia. First, the article provides a short-conceptual framework of reference. Second, it contextualizes the Movement internationally, and establishes its main features. Third, it discusses the Movement in the region of Andalusia. Fourth, it analyzes the Movement's impact on Andalusian public opinion. Conclusions are provided.
\end{abstract}

KEY WORDS: Outraged, Andalusia, social movements.

Este artículo es resultado del contrato de investigación "Elementos de continuidad y cambio en la cultura política andaluza: reconstruyendo la confianza política en Andalucía en el actual contexto de crisis", financiado por la Fundación pública andaluza Centro de Estudios Andaluces (2013). El símbolo \# remite a las etiquetas de Twitter.

** Universidad Pablo de Olavide, Facultad de Derecho, Departamento de Derecho Público, Sevilla, España. Correo electrónico: japer@upo.es 


\section{INTRODUCCIÓN}

Como ha establecido McAdam (2002: 244), al abordar los movimientos sociales (MS) no debemos considerar como unidades de análisis los propios, sino los ciclos de protesta en los que estos se insertan. Los movimientos sociales son actores no estatales que constituyen una de las principales formas colectivas de interconexión entre el poder político y la sociedad civil. Su aparición -en su sentido contemporáneo- data del siglo XIX. Se han aportado múltiples y variadas definiciones sobre los movimientos sociales (Scott 1990: 6; Giddens 2000: 645). Según Snow, Soule y Kriese se trata de

colectividades que actúan con algún grado de organización y continuidad fuera de los canales institucionales o de organización con el propósito de desafiar o defender la autoridad existente, sea de base institucional o cultural, dentro del grupo, organización, sociedad, cultura, u orden mundial del que formen parte (2004: 11).

Dentro de los movimientos sociales, nuestro marco de referencia serán los nuevos movimientos sociales (NMS) -surgidos en los años 60 del siglo XX y consolidados en la década siguiente-, donde se encuadra el Movimiento 15-M en España y en Andalucía.

Para Tarrow (1997), un ciclo de protesta es una oleada de acciones colectivas (y de reacciones ante ellas) cuya frecuencia, intensidad y manifestaciones experimentan un crecimiento seguido -en un espacio temporal relativamente próximo- de un declive, expandiendo el conflicto a la sociedad en su conjunto, desde los sectores que habitualmente presentan mayores índices de movilización, hasta los menos movilizados, e introduciendo también innovaciones en la gama de acciones colectivas. Este autor ha distinguido tres ciclos de protesta modernos. El primero de ellos transcurriría desde 1848 -aproximadamente- hasta el período de entreguerras; el segundo transcurriría, en su etapa de apogeo, entre los años 60 del siglo XX y comienzos de los años 80, período en el que surgen los nuevos movimientos sociales; y el tercer ciclo de protesta habría empezado en la segunda mitad de los años 90.

\section{CONTEXTO INTERNACIONAL Y CARACTERIZACIÓN DEL 15-M}

Aunque aún no resulta posible determinar si el actual fenómeno global de protesta y movilización, iniciado en 2010, es la continuación de ese tercer ciclo de protesta o el inicio de un cuarto ciclo, podemos afirmar que se trata de un fenómeno que quizá sea el más intenso desde mayo de 1968 (el cual se inserta dentro del segundo ciclo de protesta de Tarrow). Se trata de un conjunto heterogéneo de movimientos y protestas surgidos 
en Francia en septiembre de 2010 -y expandidos posteriormente a decenas de países de los cinco continentes- en los que se inscribe el Movimiento 15-M (en adelante, también Movimiento y 15-M) en España y en Andalucía. Su origen principal e hilo vertebrador son las repercusiones para la ciudadanía de la crisis financiera internacional iniciada en 2007. Estos movimientos y protestas atribuyen la responsabilidad de la crisis a un sistema financiero de naturaleza especulativa y carente de control gubernamental. Sin embargo, los actuales movimientos y protestas tienen como una de sus peculiaridades el que la mayoría de las reivindicaciones y propuestas que plantean se encuentran muy focalizadas en los diferentes contextos nacionales.

Podemos señalar cuatro episodios claves del actual fenómeno de movilización: (i) el surgimiento en Francia; (ii) la denominada Primavera Árabe; (iii) el Movimiento 15-M en España; y (iv) el salto de las protestas a Estados Unidos. Como hemos apuntado, situaremos el comienzo de este fenómeno en septiembre de 2010, cuando la pretensión del gobierno francés de retrasar la edad de jubilación y de aplicar importantes recortes presupuestarios provocó una huelga general y manifestaciones multitudinarias a las que se sumaron los transportistas, que presionaron al gobierno con el desabastecimiento de carburantes. Un mes después, en octubre de 2010, el autor francés S. Hessel publicó en Francia su obra ¡Indignaos!, convertida en uno de los referentes intelectuales del actual fenómeno de movilización y protesta.

En este se incardina también la Primavera Árabe, que propició la caída en 2011 de los regímenes tunecino, egipcio y libio, y la desestabilización de otros gobiernos de la región. En Túnez, en diciembre de 2010, tuvo lugar el inicio de este proceso con la denominada Revolución de los Jazmines.

En septiembre de 2011, dentro de otro ámbito geográfico, las protestas alcanzaron a Estados Unidos, de la mano del movimiento Occupy Wall Street (Bennett y Segerberg 2011: 7, 25-6). Este surgió concretamente en Manhattan, y atrajo de manera decisiva la atención de los medios de comunicación más influyentes hacia este fenómeno mundial de movilización y protesta.

\section{15-M ANDALUZ}

Más allá del contexto internacional que acabamos de esbozar, es necesario realizar un análisis complementario en clave doméstica del Movimiento 15-M en España y en Andalucía, concretamente como la continuación de un ciclo de protesta genuinamente espa- 
ñol que según Herreros (2008: 115-21) coincidiría con la primera legislatura del gobierno de Zapatero (2004-2008). Durante ese período emergieron movilizaciones por el derecho a la vivienda y contra la precarización de la vida, y, a su vez, estos movimientos generaron nuevas herramientas e instituciones, como la Red Estatal por los Derechos de los Migrantes.

Hay que aclarar que desde el surgimiento del Movimiento 15-M se han empleado en España de manera indistinta diversas denominaciones para designarlo, entre otras: Movimiento de los Indignados, Movimiento Democracia Real Ya, Movimiento Quincemayista y Movimiento 15-M. Esta última denominación -debido fundamentalmente a su simplicidad- ha sido la que finalmente se ha generalizado y consolidado en el lenguaje cotidiano. La expresión hace referencia al 15 de mayo de 2011, en el cual el Movimiento empezó a visibilizarse en España y en Andalucía, en los días previos a la celebración de las elecciones municipales y autonómicas del 22 de mayo del 2011, las cuales, en el caso de Andalucía, fueron únicamente municipales.

El domingo 15 de mayo del 2011, siete días antes de la celebración de dichas elecciones, unos 130.000 ciudadanos - principal pero no exclusivamente jóvenes- se manifestaron en más de 50 ciudades españolas bajo el lema unitario Democracia Real Ya (DRY). Se trató de una acción colectiva, no organizada ni auspiciada por partidos políticos, sindicatos u otras organizaciones políticas tradicionales, sino por plataformas ad hoc que operaban principalmente en internet y sus redes sociales (Anduiza, Cristancho y Sabucedo 2011: 2), bajo la etiqueta (hashtag) \#15mani. La importancia de las nuevas tecnologías de la información en la generación y coordinación de este movimiento, tuvo igualmente su reflejo en la repercusión internacional de estas movilizaciones.

Los partidarios de las mismas hicieron un amplio uso en el ciberespacio del lema Spanish Revolution -acuñado por The Washington Post-, como estrategia para conectar con una audiencia internacional, pero igualmente como un marco cognitivo que ligaba las protestas en España con los cambios revolucionarios que habían tenido lugar en el mundo árabe.

La plataforma central, integrada por más de 400 organizaciones de todo el Estado bajo el lema ¡Democracia Real YA! (DRY), logró convocar a miles de andaluces, sobre todo en las ocho capitales de provincia y en los grandes municipios. A estas manifestaciones les siguieron acampadas en plazas de las principales ciudades, en las cuales tuvieron lugar asambleas diarias y la creación de diferentes comités ciudadanos que continuaron funcionando durante más de un mes. Las acampadas fueron iniciadas por varios centenares de manifestantes que decidieron pasar la noche en las plazas. Sin embargo, no fueron 
promovidas ni apoyadas por la plataforma DRY, sino que se organizaron mediante las redes sociales (Anduiza, Cristancho y Sabucedo 2011: 2), fundamentalmente Twitter, bajo la etiqueta \#acampadasol y posteriormente \#acampada [nombre de la ciudad].

La primera acampada surgió en la Puerta del Sol de Madrid, y a la iniciativa se sumaron al día siguiente ciudades como Barcelona y Valencia, y, en Andalucía, Sevilla y Málaga. De hecho, desde el comienzo, las ciudades de Sevilla, Granada y Málaga han jugado un papel fundamental en la expansión y vertebración del Movimiento 15-M a nivel nacional. Las acampadas más numerosas en Andalucía tuvieron lugar en estas tres capitales de provincia y en las cinco restantes: Cádiz, Huelva, Almería, Córdoba y Jaén.

Los días previos al 15 de mayo del 2011 la atención prestada por los medios de comunicación a la convocatoria había sido escasa (tampoco previamente los medios habían informado sobre el proceso de creación de la plataforma DRY). Pero, a raíz del éxito de la convocatoria, durante los días inmediatamente posteriores a esta fecha los medios de comunicación situaron su foco en la cobertura de todo lo relacionado con el Movimiento. Así, el extraordinario eco logrado por este, incluido su momento de auge en las horas anteriores a la celebración de las elecciones, no se explica sin la cobertura dada al mismo por los medios de comunicación, en un proceso en que estos y el 15-M se retroalimentaban mutuamente.

Los diferentes medios abordaron informaciones acerca de las protestas dentro de sus respectivos encuadres (framing) editoriales (Scheufele 1999). De ese modo, la incertidumbre acerca del impacto, naturaleza y desarrollo de las protestas fue procesada a través de interpretaciones que priorizaban unos elementos sobre otros, para que la noticia tuviese un significado acorde a las preferencias y prejuicios de sus respectivas audiencias. Algunos medios se centraron en el efecto regenerador que las movilizaciones podían tener sobre el sistema democrático y la estructura de partidos en España. Según esto, la ciudadanía, ante la incapacidad y desconexión social de los partidos políticos tradicionales, había conseguido incluir en la agenda política una serie de demandas y prioridades de actuación que hasta el momento no habían sido convenientemente atendidas por la clase política. Otros medios enfatizaron el carácter ilegítimo de unas protestas que, a través de la ocupación no autorizada del espacio público, pretendían romper la normalidad en el desarrollo de la campaña electoral con el objetivo último de alterar el resultado de los comicios.

Sin embargo, los diferentes enfoques tuvieron como nexo de unión plantear la interrogante sobre si las movilizaciones tendrían impacto en los resultados electorales y 
qué fuerzas políticas resultarían beneficiadas y cuáles perjudicadas, cuestión que abordaremos en el epígrafe 3.

En cuanto a las reivindicaciones y propuestas del $15-\mathrm{M}$, el catedrático de ciencia política Ramón Cotarelo (2011) ha ofrecido una caracterización sintética del movimiento, señalando que se trataba de:

un levantamiento pacífico de ciudadanos, principalmente jóvenes pero no sólo ellos, hartos de padecer la crisis y la forma de gestionarla del sistema político. Y un movimiento espontáneo, nacido en la nueva esfera pública virtual cuya eficacia práctica es indudable. Es rápida, es flexible, recibe mucha información en tiempo real y se adapta a las circunstancias. Es decir, sobrevive [...]. Es una explosión política pacífica extraparlamentaria que se dirige contra el sistema en su conjunto, el nacional y el internacional, contra la banca, los empresarios, los sindicatos, los partidos, los políticos, las instituciones, los medios de comunicación (Palinuro 2011).

A continuación reproducimos de manera íntegra uno de los documentos difundidos por el Movimiento 15-M, que sintetiza todas o la mayoría de sus propuestas:

\section{Documento 1. Propuestas del 15-M}

\section{ELIMINACIÓN DE LOS PRIVILEGIOS DE LA CLASE POLÍTICA:}

- Control estricto del absentismo de los cargos electos en sus respectivos puestos. Sanciones específicas por dejación de funciones.

- Supresión de los privilegios en el pago de impuestos, los años de cotización y el monto de las pensiones. Equiparación del salario de los representantes electos al salario medio español, más las dietas necesarias indispensables para el ejercicio de sus funciones.

- Eliminación de la inmunidad asociada al cargo. Imprescriptibilidad de los delitos de corrupción.

- Publicación obligatoria del patrimonio de todos los cargos públicos.

- Reducción de los cargos de libre designación. 


\section{CONTRA EL DESEMPLEO:}

- Reparto del trabajo fomentando las reducciones de jornada y la conciliación laboral hasta acabar con el desempleo estructural (es decir, hasta que el desempleo descienda por debajo del 5 por ciento).

- Jubilación a los 65 y ningún aumento de la edad de jubilación hasta acabar con el desempleo juvenil.

- Bonificaciones para aquellas empresas con menos de un 10 por ciento de contratación temporal.

- Seguridad en el empleo: imposibilidad de despidos colectivos o por causas objetivas en las grandes empresas mientras haya beneficios, fiscalización a las grandes empresas para asegurar que no cubren con trabajadores temporales empleos que podrían ser fijos.

- Restablecimiento del subsidio de $426 €$ para todos los parados de larga duración. 3. DERECHO A LA VIVIENDA:

- Expropiación por el Estado de las viviendas construidas en stock que no se han vendido para colocarlas en el mercado en régimen de alquiler protegido.

- Ayudas al alquiler para jóvenes y todas aquellas personas de bajos recursos.

- Que se permita la dación en pago de las viviendas para cancelar las hipotecas. 4. SERVICIOS PÚBLICOS DE CALIDAD:

- Supresión de gastos inútiles en las Administraciones Públicas y establecimiento de un control independiente de presupuestos y gastos.

- Contratación de personal sanitario hasta acabar con las listas de espera.

- Contratación de profesorado para garantizar la ratio de alumnos por aula, los grupos de desdoble y los grupos de apoyo.

- Reducción del coste de matrícula en toda la educación universitaria, equiparando el precio de los posgrados al de los grados.

- Financiación pública de la investigación para garantizar su independencia.

- Transporte público barato, de calidad y ecológicamente sostenible: restablecimiento de los trenes que se están sustituyendo por el AVE con los precios originarios, abaratamiento de los abonos de transporte, restricción del tráfico rodado privado en el centro de las ciudades, construcción de carriles bici.

- Recursos sociales locales: aplicación efectiva de la Ley de Dependencia, redes de cuidadores locales municipales, servicios locales de mediación y tutelaje. 


\section{CONTROL DE LAS ENTIDADES BANCARIAS:}

- Prohibición de cualquier tipo de rescate o inyección de capital a entidades bancarias: aquellas entidades en dificultades deben quebrar o ser nacionalizadas para constituir una banca pública bajo control social.

- Elevación de los impuestos a la banca de manera directamente proporcional al gasto social ocasionado por la crisis generada por su mala gestión.

- Devolución a las arcas públicas por parte de los bancos de todo capital público aportado.

- Prohibición de inversión de bancos españoles en paraísos fiscales.

- Regulación de sanciones a los movimientos especulativos y a la mala praxis bancaria.
6. FISCALIDAD:

- Aumento del tipo impositivo a las grandes fortunas y entidades bancarias.

- Eliminación de las Sociedades de Inversión de Capital Variable (SICAV).

- Recuperación del Impuesto sobre el Patrimonio.

- Control real y efectivo del fraude fiscal y de la fuga de capitales a paraísos fiscales.

- Promoción a nivel internacional de la adopción de una tasa a las transacciones internacionales (tasa Tobin). 7. LIBERTADES CIUDADANAS Y DEMOCRACIA PARTICIPATIVA:

- No al control de internet. Abolición de la Ley Sinde.

- Protección de la libertad de información y del periodismo de investigación.

- Referéndums obligatorios y vinculantes para las cuestiones de gran calado que modifican las condiciones de vida de los ciudadanos.

- Referéndums obligatorios para toda introducción de medidas dictadas desde la Unión Europea.

- Modificación de la Ley Electoral para garantizar un sistema auténticamente representativo y proporcional que no discrimine a ninguna fuerza política ni voluntad social, donde el voto en blanco y el voto nulo también tengan su representación en el legislativo.

- Independencia del Poder Judicial: reforma de la figura del Ministerio Fiscal para garantizar su independencia, no al nombramiento de miembros del Tribunal Constitucional y del Consejo General del Poder Judicial por parte del Poder Ejecutivo.

- Establecimiento de mecanismos efectivos que garanticen la democracia interna en los partidos políticos.

\section{REDUCCIÓN DEL GASTO MILITAR}

Fuente: ¡Democracia Real YA! (2011). 
El Estudio General de Opinión Pública de Andalucía (EGOPA), correspondiente al otoño de 2011 -publicado por el Centro de Análisis y Documentación Política y Electoral de Andalucía (CADPEA)-, situaba en 5,50 el grado de acuerdo medio de los ciudadanos andaluces con las demandas planteadas por el Movimiento 15-M (Gráfico No 1), en una escala de 0 (totalmente en desacuerdo) a 10 (totalmente de acuerdo).

\section{Gráfico No 1}

Grado de acuerdo medio con las demandas del Movimiento 15M (Media = 5,50)

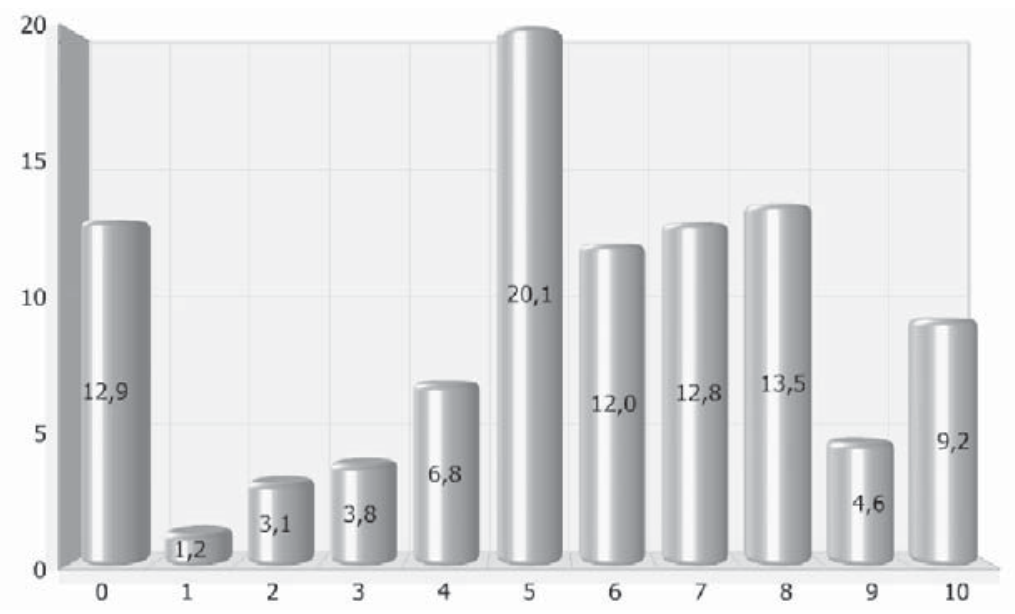

Fuente: EGOPA (CADPEA, 2012).

En cuanto a la evolución del Movimiento 15-M, un mes después de su surgimiento, concretamente el 19 de junio de 2011 (19-J), la plataforma DRY logró convocar manifestaciones aún más multitudinarias que las del 15-M, bajo un nuevo lema: Toma la calle! como protesta por el Pacto por el Euro adoptado por la Comisión Europea, con el fin de realizar reformas destinadas a mejorar la fortaleza fiscal y la competitividad. Miles de andaluces participaron en estas manifestaciones. La más multitudinaria de todas tuvo lugar en la ciudad de Sevilla: según el Movimiento, logró reunir a casi 7.000 ciudadanos. Después de la celebración de la manifestación, en Sevilla -al igual que en otras ciudades, como Málaga-, el 15-M levantó la acampada.

Cuatro meses después, el 15 de octubre de 2011 (15-O), coincidiendo con el quinto mes de existencia del Movimiento 15-M en España, en el marco de una convocatoria a nivel mundial bajo el lema "Unidos por un cambio global", tuvieron lugar en numerosos 
municipios andaluces manifestaciones más multitudinarias que las celebradas el 19-J. En las ocho capitales andaluzas se congregaron aproximadamente: 50.000 en Sevilla y 4.000 en Huelva (según los convocantes); 4.000 en Málaga, 3.500 en Granada, 3.000-4.000 en Almería, 3.000 en Córdoba, 1.100-1.400 en Jaén, y 400 en Cádiz (según fuentes policiales). A estas cifras hay que sumar un número indeterminado de manifestantes en el resto de los municipios de Andalucía.

La semana posterior a la celebración en España de las elecciones generales (20 de noviembre de 2011), en concreto el 26 y 27 de noviembre de 2011, se celebró el IV Encuentro Estatal del 15M, bajo el lema "De la protesta a la propuesta y de la propuesta al compromiso", el cual pudo seguirse en internet a través de la herramienta Mumble. El lugar de celebración fue el municipio sevillano de Marinaleda, gobernado por el comunista J. M. Sánchez Gordillo.

El IV Encuentro estuvo abierto a la participación de todas las asambleas, ciudades, pueblos, barrios, comisiones y grupos de trabajo, y ciudadanos. Según los organizadores, sus propósitos fundamentales eran: (i) seguir mejorando las formas internas de organización y de comunicación del Movimiento 15-M; (ii) continuar compartiendo las diferentes visiones, experiencias y propuestas surgidas en torno a sus asambleas por todo el Estado; (iii) seguir construyendo de forma horizontal y abierta las formas de lucha colectivas; y (iv) continuar actuando en pos de los objetivos comunes a todos los integrantes del 15-M, planteados sobre la base del programa del Movimiento.

Durante el último mes de 2011 tuvieron lugar nuevos actos de protesta en Andalucía. En concreto, el 18 de diciembre de 2011, víspera de la constitución de las Cortes Generales surgidas tras las elecciones del 20 de noviembre de 2011, tuvo lugar en Sevilla una manifestación que reivindicaba un sistema electoral justo y proporcional, en el marco de la campaña ;Una persona, un voto!, que DRY asegura mantendrá durante la legislatura 2011-2015. En este sentido, nuevamente según el EGOPA correspondiente al otoño de 2011, el 11 por ciento de los andaluces encuestados aseguraba haber participado a lo largo de 2011 en al menos una de las actividades de movilización del 15-M (Gráfico No 2). 


\begin{abstract}
Gráfico No 2
¿Ha participado Ud. en alguna de las movilizaciones del 15-M?
\end{abstract}

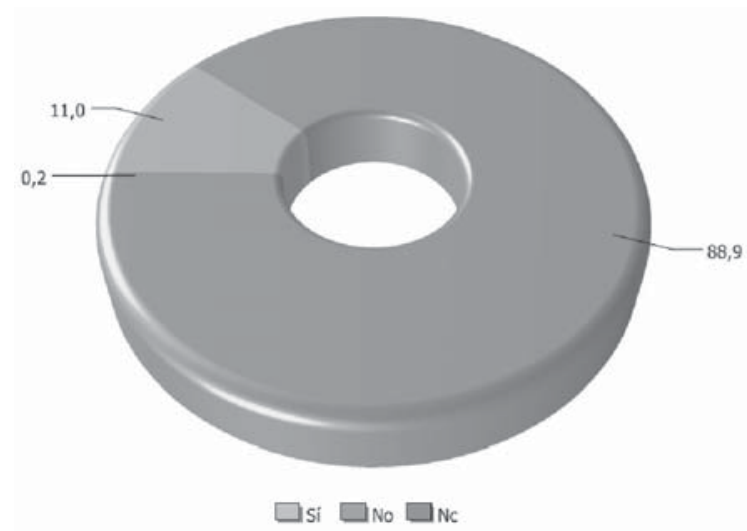

Fuente: EGOPA (CADPEA 2012).

De hecho, el Movimiento 15-M ya tenía previsto a finales de 2011 mantener sus estructuras organizativas y continuar con el desarrollo de sus actividades a lo largo de 2012; entre ellas, la usurpación de bienes inmuebles y la obstaculización de la ejecución de órdenes de desahucio que considera injustas. Durante 2012, las Fuerzas y Cuerpos de Seguridad del Estado (FyCSE) procedieron a la realización de desalojos de bienes inmuebles ocupados por miembros del Movimiento, y actualmente varios continúan ocupados en diversos municipios de Andalucía.

Durante parte de mayo de 2012, concretamente a partir del 12, el 15-M desarrolló un programa de actividades -con " $12 \mathrm{M} 15 \mathrm{M}$ " como uno de sus principales lemas- para conmemorar su primer aniversario de existencia y realizar un balance de su actuación durante este primer año. El programa incluía manifestaciones, asambleas, concentraciones, pancartadas, conferencias, mesas redondas, discursos, charlas, debates, conciertos de música, proyecciones de películas, videoforums, talleres, representaciones teatrales y fiestas. Concretamente el mencionado 12 de mayo, el Movimiento 15-M congregó en Andalucía a miles de ciudadanos en decenas de manifestaciones, fundamentalmente en sus ocho capitales de provincia, en el marco de una nueva movilización a nivel mundial (\#12MGLOBAL), similar a la que tuvo lugar cinco meses después, el 13 de octubre de 2012 (\#13O o \#GLOBALNOISE). 
Actualmente se encuentra en marcha el macroproyecto cultural audiovisual $15 \mathrm{M}$. cc --vinculado al Movimiento- en el que las andaluzas Sevilla y Málaga están jugando un papel primordial a nivel estatal (respectivamente, con los programas 15MSevilla. cc y 15MMálaga.cc, junto a Madrid.15M.cc, Audio.15M.cc y Papers.15M.cc). El objetivo principal del macropoyecto colaborativo $15 \mathrm{M} . c c$ es facilitar el mayor número posible de narraciones en torno al 15-M, y actualmente incluye los siguientes tres proyectos: el banco de ideas, una web de almacenamiento y consulta de materiales audiovisuales -con licencia copyleft- relacionados con el Movimiento 15-M; la 15Mpedia, una enciclopedia libre sobre el Movimiento similar a la Wikipedia; y el WikiLibro, que pretende que un armazón preestablecido de ideas vaya siendo desarrollado de manera colaborativa.

\section{IMPACTO SOBRE LA OPINIÓN PÚBLICA ANDALUZA}

Se puede afirmar que la influencia del Movimiento 15-M sobre el comportamiento electoral de los andaluces en las elecciones municipales del 22 de mayo de 2011 y en las generales del 20 de noviembre de 2011 fue, en términos generales, escasa.

Comencemos por las elecciones municipales de mayo. Según el mencionado EGOPA, correspondiente esta vez al verano de 2011, el 85 por ciento de los andaluces encuestados aseguraba que el grado de influencia ejercido por el Movimiento 15-M sobre su comportamiento electoral en dichas elecciones había sido ninguno o poco. Si se desagrega dicho porcentaje, se observa que el 79,5 por ciento aseguraba que la influencia había sido nula, y el 5,5 por ciento que la influencia había sido poca. Solo el 11,7 por ciento aseguraba que la influencia del Movimiento 15-M sobre su comportamiento electoral había sido bastante (6,8 por ciento) o mucha (4,9 por ciento) (Gráfico No 3 ). 


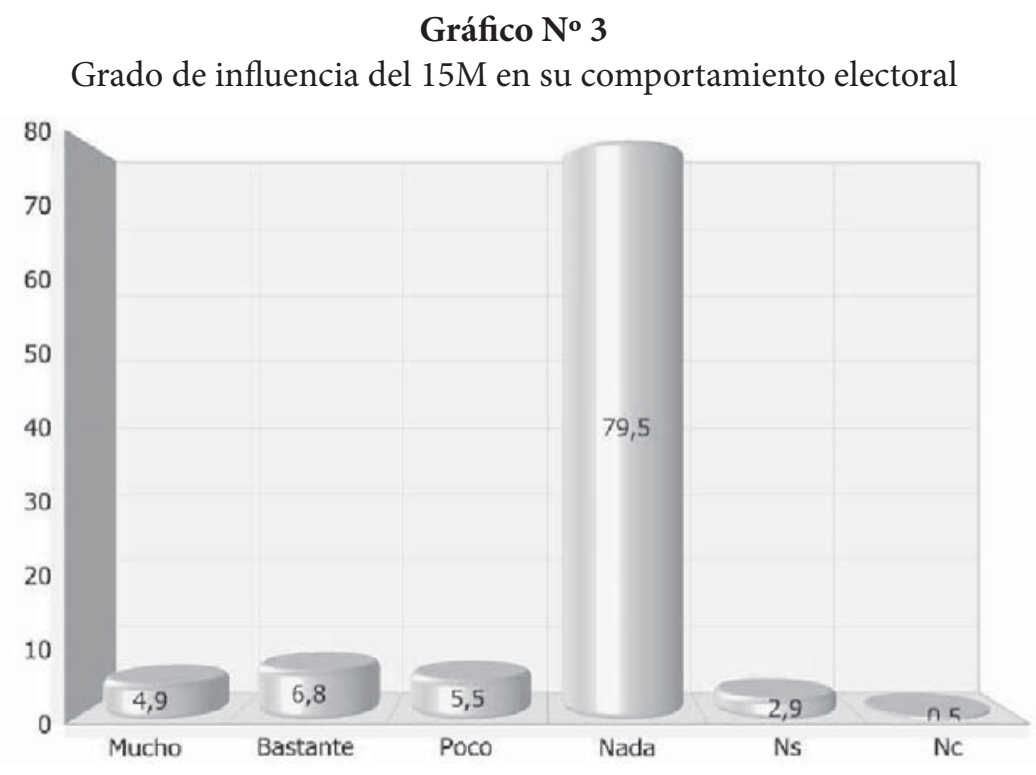

Fuente: EGOPA (CADPEA 2011).

Además, como refleja el Gráfico No 4, dentro del reducido porcentaje de ciudadanos que reconocía que el 15-M había ejercido algún grado de influencia sobre su comportamiento electoral (poco, bastante y mucho: 17,2 por ciento), el mayor porcentaje correspondía al de quienes afirmaban que el Movimiento les había animado a votar al partido político al que siempre votan (33,4 por ciento). Solo el 20,7 por ciento afirmaba que el 15-M había contribuido a cambiar el sentido de su voto, animándole a votar a un partido que no era su preferido o al que no había votado anteriormente. Y únicamente el 14,9 por ciento aseguraba que el 15-M le había generado un efecto desmovilizador, animándole a no votar, mientras que solo el 10,5 por ciento afirmaba que el Movimiento le había animado a emitir un voto blanco o un voto nulo. 


\section{Gráfico No 4}

Influencia del Movimiento $15 \mathrm{M}$ en...
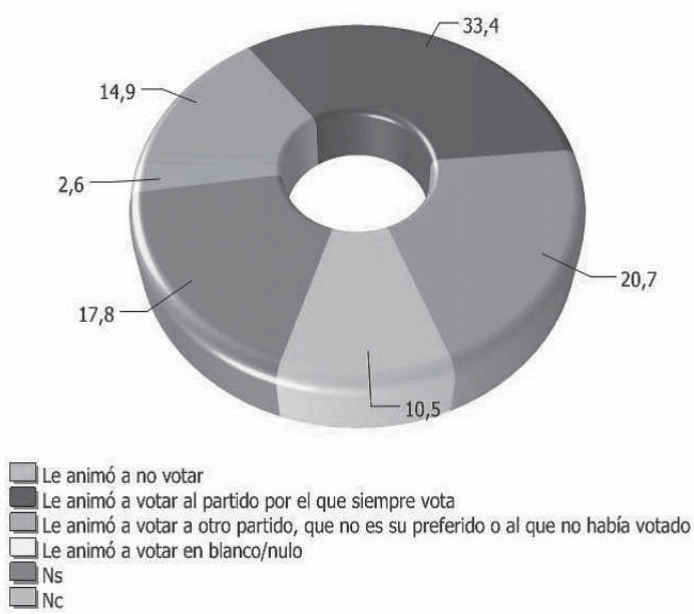

Fuente: EGOPA (CADPEA 2011).

En cuanto a las elecciones generales de noviembre, los datos del EGOPA, correspondiente al otoño de 2011, arrojan unos datos bastante similares a los de las elecciones municipales de mayo, como puede observarse en el Gráfico No 5 .

\section{Gráfico No 5}

Influencia del Movimiento 15M en su comportamiento el 20 de noviembre de 2011

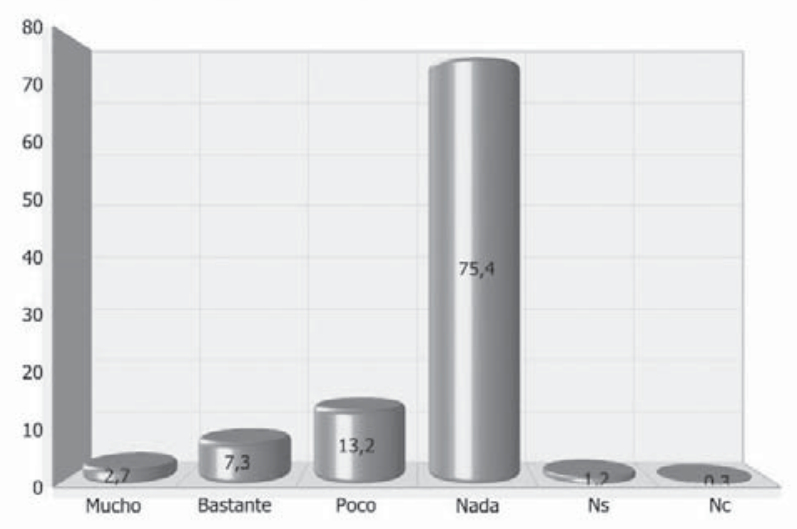

Fuente: EGOPA (CADPEA 2012). 
Como se puede comprobar, el 88,6 por ciento de los andaluces encuestados aseguraba que el grado de influencia ejercido por el Movimiento 15-M sobre su comportamiento electoral en las elecciones generales había sido ninguno o poco. Si se desagrega dicho porcentaje, se observa que el 75,4 por ciento aseguraba que la influencia había sido nula, y el 13,2 por ciento que la influencia había sido poca. Solo el 10 por ciento aseguraba que la influencia del Movimiento 15-M sobre su comportamiento electoral había sido bastante ( 7,3 por ciento) o mucha ( 2,7 por ciento).

\section{Gráfico No 6}

Influencia del Movimiento $15 \mathrm{M}$ en...
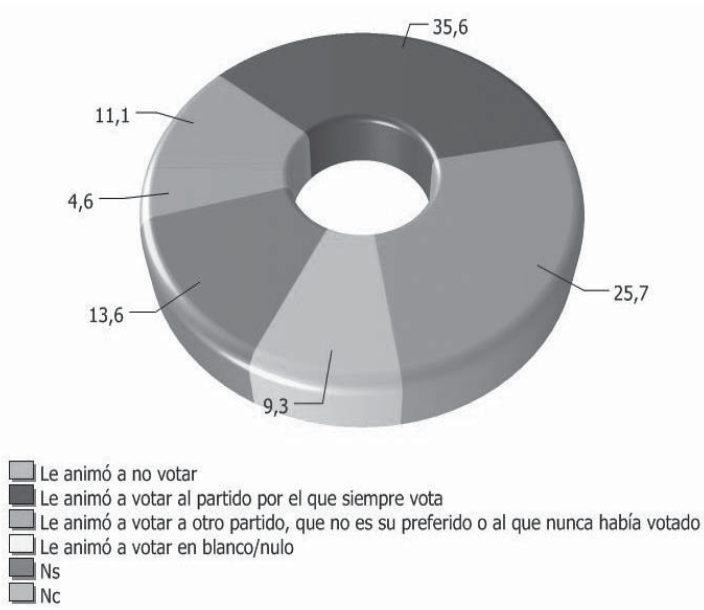

Fuente: EGOPA (CADPEA 2012).

También los datos referentes al tipo de influencia del 15-M en ambas citas electorales son bastante similares, como refleja el Gráfico $\mathrm{N}^{\circ} 6$. Así, dentro del reducido porcentaje de ciudadanos que reconocía que el 15-M había ejercido algún grado de influencia sobre su comportamiento electoral (poco, bastante y mucho: 23,2 por ciento), el mayor porcentaje correspondía al de quienes afirmaban que el Movimiento les había animado a votar al partido político al que siempre votan (35,6 por ciento). Solo el 25,7 por ciento afirmaba que el 15-M había contribuido a cambiar el sentido de su voto, animándole a votar a un partido que no era su preferido o al que no había votado anteriormente. Y únicamente el 11,1 por ciento aseguraba que el Movimiento le había generado un efecto desmovilizador, animándole a no votar, mientras que solo el 9,3 por ciento afirmaba que el 15-M le había animado a emitir un voto blanco o un voto nulo. 


\section{CONCLUSIONES}

El presente artículo exploratorio ha pretendido realizar una aproximación inicial al origen, la evolución inmediata y los efectos del Movimiento 15-M, concretamente en Andalucía. En primer lugar, se ha establecido un breve marco teórico-conceptual de referencia. En segundo lugar, se ha contextualizado el Movimiento en el plano internacional, y se han establecido sus principales características. En tercer lugar se ha abordado el Movimiento en el ámbito andaluz. Y, en cuarto lugar, se ha analizado su impacto sobre la opinión pública andaluza.

Aunque aún no resulta posible determinar si el actual fenómeno global de protesta y movilización, iniciado en 2010, es la continuación del tercer ciclo de protesta de Tarrow o el comienzo de un cuarto ciclo, podemos afirmar que se trata de un fenómeno que quizá sea el más intenso desde mayo de 1968 (el cual se inserta dentro del segundo ciclo de protesta de Tarrow). Se trata de un conjunto heterogéneo de movimientos y protestas surgidos en Francia en septiembre de 2010 -y expandidos posteriormente a decenas de países de los cinco continentes- en los que se inscribe el Movimiento 15-M en España y en Andalucía. Su origen principal e hilo vertebrador son las repercusiones para la ciudadanía de la crisis financiera internacional iniciada en 2007. Estos movimientos y protestas atribuyen la responsabilidad de la crisis a un sistema financiero de naturaleza especulativa y carente de control gubernamental. Sin embargo, los actuales movimientos y protestas tienen como una de sus peculiaridades el que la mayoría de las reivindicaciones y propuestas que plantean se encuentran muy focalizadas en los diferentes contextos nacionales.

Por ello en el caso de España y de Andalucía hemos realizado un análisis complementario en clave doméstica del Movimiento 15-M, concretamente como la continuación de un ciclo de protesta genuinamente español que según Herreros (2008: 115-21) coincidiría con la primera legislatura del gobierno de Zapatero (2004-2008). Durante ese período emergieron movilizaciones por el derecho a la vivienda y contra la precarización de la vida, $y$, a su vez, los movimientos sociales fueron generando nuevas herramientas e instituciones, como la Red Estatal por los Derechos de los Migrantes.

El 15-M demanda la supresión de los privilegios de la clase política, la lucha contra el desempleo, la potenciación del derecho a la vivienda, el control de las entidades bancarias y financieras, la elevación de los impuestos, el aumento de las libertades ciudadanas y la mejora de la democracia participativa, y la reducción del gasto militar. Aunque el EGOPA correspondiente al otoño de 2011 situaba en 5,50 el grado de acuerdo medio de 
los ciudadanos andaluces con las demandas planteadas por el Movimiento 15-M (Gráfico 1), en una escala de 0 (totalmente en desacuerdo) a 10 (totalmente de acuerdo), se puede afirmar que la influencia del Movimiento sobre el comportamiento electoral de los andaluces en las elecciones municipales del 22 de mayo de 2011 y en las generales del 20 noviembre de 2011 fue, en términos generales, escasa.

\section{REFERENCIAS}

1. Anduiza, Eva, Camilo Cristancho y José Sabucedo. 2011. The political protest of the outraged in Spain: what's new? Mimeo.

2. Bennett, W. Lance y Alexandra Segerberg. 2011. "The Logic of Connective Action: Digital Media and the Personalization of Contentious Politics." En Simposio A Decade in Internet Time. Oxford: Universidad de Oxford.

3. Centro de Análisis y Documentación Política y Electoral de Andalucía (CADPEA). 2011. Estudio General de Opinión Pública de Andalucía (EGOPA). Granada: CADPEA.

4. ----- 2012. Estudio General de Opinión Pública de Andalucía (EGOPA). Granada: CADPEA.

5. ¡Democracia Real YA! 2011. “Propuestas”. Bizkaia: ¡Democracia Real YA! Consultado el 12 de noviembre de 2013 (http://bizkaia.democraciarealya.es/propuestas/).

6. Fernández de Rota, A. 2011. "Las políticas de la multitud: De la antropología reflexiva al movimiento por una democracia real." Etnicex: revista de estudios etnográficos 2: 5376.

7. Giddens, Anthony. 2000. Sociología. Madrid: Alianza.

8. Herreros, Tomás. 2008. "Entender los movimientos sociales desde otras miradas." Pp. 97-124 en La acción política desde la comunidad: análisis y propuestas, coordinado por P. Heras i Trias. Barcelona: GRAÓ.

9. McAdam, Doug. 2002. "Movimientos iniciadores y derivados: procesos de difusión en los ciclos de protesta.” Pp. 243-70 en Protesta Social, editado por M. Traugott. Barcelona: Hacer. 
10. Palinuro. 2011. "Esto es sólo el comienzo..." Madrid: Ramón Cotarelo. Consultado el 6 de octubre de 2012 (http://cotarelo.blogspot.com/2011/05/esto-es-solo-el-comienzo.html).

11. Scheufele, Dietram A. 1999. "Framing as a theory of media effects." Journal of Communication 49 (1): 103-22.

12. Scott, Allan. 1990. Ideology and the New Social Movements. Londres: Unwin Hyman.

13. Snow, David A., Sarah A. Soule y Hanspeter Kriesi. 2004. "Mapping the Terrain." Pp. 3-16 en The Blackwell Companion to Social Movements, editado por David A. Snow, Sarah A. Soule y Hanspeter Kriesi. Oxford, UK: Blackwell Publishing.

14. Tarrow, Sydney. 1997. El poder en movimiento: los movimientos sociales, la acción colectiva y la política. Madrid: Alianza. 